\title{
Impactos del cambio climático en el patrimonio territorial de los destinos turísticos: el caso del Cantón de Carrillo, Costa Rica
}

Pablo Miranda Álvarez

\section{SciELO Books / SciELO Livros / SciELO Libros}

MIRANDA ÁLVAREZ, P. Impactos del cambio climático en el patrimonio territorial de los destinos turísticos: el caso del Cantón de Carrillo, Costa Rica. In: MARAFON, G. J., FACCIOLI, M., and SÁNCHEZ, M. A., ed. Patrimônio, território e turismo no Brasil, Costa Rica e Itália [online]. Rio de Janeiro: EDUERJ, 2020, pp. 69-93. ISBN: 978-65-00-03032-7.

https://doi.org/10.7476/9786500030327.0004.

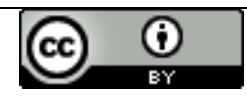

All the contents of this work, except where otherwise noted, is licensed under a Creative Commons Attribution 4.0 International license.

Todo o conteúdo deste trabalho, exceto quando houver ressalva, é publicado sob a licença Creative Commons Atribição 4.0.

Todo el contenido de esta obra, excepto donde se indique lo contrario, está bajo licencia de la licencia Creative Commons Reconocimento 4.0. 


\section{Impactos del cambio climático en el patrimonio territorial de los destinos turísticos: el caso del Cantón de Carrillo, Costa Rica}

Pablo Miranda Álvarez

\section{Introducción}

El cantón de Carrillo, uno de los principales destinos de sol y playa en Costa Rica, se encuentra localizado en el Pacífico Norte del país y es afectado constantemente por eventos asociados al cambio climático como los fenómenos del Niño y la Niña. Ejemplo de ello fue la sequía causada por el fenómeno del Niño entre los años 2013 y 2015, la más severa de los últimos cincuenta años, que afectó los diferentes componentes del patrimonio territorial como destino turístico y sus comunidades locales.

Con el fin de determinar los impactos causados en el patrimonio territorial por el cambio climático durante este periodo, se realiza una clasificación de los usos del suelo en el año 2017 y la caracterización de los principales elementos de la actividad turística del cantón mediante los cuales se identifican dichos impactos en las comunidades del cantón de Carrillo y su aporte para la planificación territorial de la actividad turística de la zona.

El patrimonio territorial como base para el desarrollo de los destinos turísticos y su relación con el cambio climático

La definición de patrimonio ha evolucionado en las últimas décadas, pasando de la valoración estética de la singularidad monumental de un elemento a un concepto más amplio que incorpora elementos tangible e intangibles históricamente excluidos como los usos del suelo y las tradiciones orales relacionadas 
a la pérdida de saberes tradicionales que por el momento histórico que vivimos son socialmente bien valorados (Doctor, 2011).

Aunque tradicionalmente el estudio del patrimonio (natural y cultural) se ha realizado de forma aislada, la Geografía como ciencia integradora a partir del abordaje del espacio geográfico como objeto de estudio, ha logrado analizarlo desde una visión holística la cual es reflejada por medio del patrimonio territorial, el cual es definido por Trujillo (2013) como:

"el espacio geográfico como objeto de interés patrimonial en tanto que resultado de la permanente interacción entre hombre y medio y que insta a observar, interpretar y gestionar de manera unitaria el conjunto de los componentes, tanto naturales y paisajísticos que lo conforman" (p. 174).

Esta visión integral del espacio por parte de la sociedad actual, mostrada por Valcárcel (1998), da como resultado la puesta en valor de los recursos naturales y culturales, desde el punto de vista comercial del uso del suelo original y la de sus posibles nuevos usos, entre ellos el turismo (figura 1):

En el caso del territorio como patrimonio, ese valor de uso procede tanto del "uso" directo, residencial, productivo, recreativo o de otro tipo, estimulado por la existencia de una demanda social solvente para tales elementos, como el "uso" indirecto, motivado por la valoración social del "patrimonio" territorial como tal, convertido este como objeto de consumo (p. 46).

Figura 1: Conformación turística del patrimonio territorial

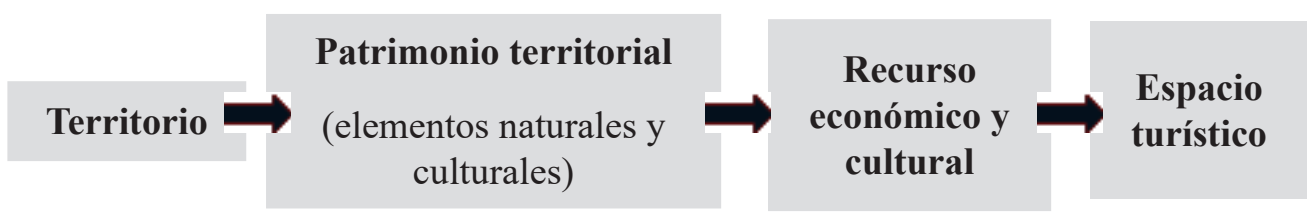

Fuente: elaboración propia a partir de Valcárcel, 1998

El turismo, como uno de los fenómenos contemporáneos económica, social y culturalmente más relevantes a nivel mundial, se fundamenta en el pa- 
trimonio territorial de las comunidades locales, lo cual lo ha convertido en una opción para su desarrollo, aunque no deja de ser una amenaza para su población en el caso de no ser bien planificada tal y como lo indica Doctor (2011):

El paisaje/patrimonio territorial es actualmente percibido (Vahí, 2011) como un eficaz herramienta de desarrollo local - en el sentido señalado por autores como Arocena $(2001)$ o Klein $(2006,303)$ de generar o reforzar «dinámicas sistémicas de escala local» -, de manera más conspicua cuando se integran en ellos los componentes naturales. En ese sentido, la promoción de una actividad turística basada en dichos recursos y planteada con criterios de sostenibilidad constituye un eje central de las políticas de desarrollo territorial, especialmente en ámbitos rurales desfavorecidos, precisamente aquéllos en los que el propio Klein alerta de la dificultad de generar dinámicas de desarrollo. Esta orientación turística del patrimonio territorial comporta, no obstante, dos riesgos. En primer lugar, y a partir de lo expresado por Ortega $(2008,52)$, el de la pérdida de sus valores propios, sustituidos por aquellos otros que se demandan como objeto de consumo, proceso que ha sido nombrado de diversas formas. En segundo, el olvido de los intereses y objetivos de la población residente (p. 278).

Por las razones anteriores, el destino turístico (por su carácter complejo) se convierte en un elemento clave para el desarrollo local, el cual debe de ser territorial y administrativamente planificado desde una perspectiva sostenible con el fin de minimizar los impactos indicados al implantarse la actividad turística. El destino turístico puede adoptar numerosas definiciones que dependen de las perspectivas y disciplinas de los investigadores que lo estudian. De una forma muy simple, puede definirse como el lugar donde se realizan las actividades turísticas, sin embargo, esta definición minimiza y deja de lado las relaciones turísticas y no turísticas que se presentan en él, las cual son cruciales para el desarrollo de la actividad turística. Según Antón (1998), la dinámica y evolución de un espacio de destino turístico está fuertemente condicionada por: los componentes territoriales, económicos y sociales que lo caracterizan, por el contexto económico de la región donde se localiza y por las tendencias económicas generales que afectan a la oferta y la demanda a nivel global.

Por tal razón, Barrado (2004) propone una definición desde una perspectiva sistémica que permite incorporar las relaciones complejas entre sus componentes. Así, para este investigador, el destino turístico: 
Debe ser entendido como un subsistema formado por elementos espaciales (recursos territoriales, infraestructuras, etc), administrativos (legislación, políticas) y productivos (factores y recursos de producción, agentes, inversión, etc); así como por el conjunto de sus interrelaciones y los efectos que producen, que son fundamentalmente sectoriales (unos bienes y servicios producidos y consumidos in situ) y geográficos (nuevas realidades paisajísticas, cambios en las relaciones territoriales previas, precepción del territorio, imágenes, etc) (p. 56).

De forma general, y aunque no lo enuncien explícitamente, las anteriores definiciones toman el destino turístico como un sistema abierto; en ellas pueden identificarse componentes comunes como: límites físicos y administrativos, recursos naturales y culturales, infraestructura general, productos turísticos y una administración pública y privada que gestione los componentes anteriores ante la demanda, especialmente para la población local que, en la mayoría de los casos, reside de forma permanente en ese territorio y es afectada directamente por los impactos naturales, económicos y sociales tanto positivos como negativos que se presenten en la actividad turística en sus diferentes escalas.

Por estas relaciones como sistema complejo, la Geografía del Turismo, desde su análisis espacial, considera el clima y el tiempo atmosférico como recursos naturales de la experiencia turística, que influyen en la planificación y desarrollo de la actividad turística, lo que genera repercusiones directas en la rentabilidad de la misma, por razones ambientales como la configuración de procesos naturales como flora y fauna, cuerpos de agua, procesos erosivos y distribución de enfermedades, elementos clave en el desarrollo del destino turístico; además, están los socioeconómicos asociados con la estacionalidad, la pérdida de infraestructuras por eventos extremos y satisfacción de visitantes así como de planificación de las redes de comunicación y transporte, edificaciones que mejoren el nivel de confort de los visitantes, la programación de actividades, el desarrollo de nuevos productos turísticos y la adaptación de los existentes, elementos a considerar dentro de las acciones de adaptación y mitigación ante el cambio climático.

Por lo tanto, el clima puede considerarse como soporte y factor de localización de las actividades turísticas, como recurso turístico natural y como atractivo turístico al ser parte del producto turístico y contribuyendo a que los turistas realicen sus actividades con seguridad y confort (Esteban et al, 2007), 
variables a considerar dentro de los procesos de ordenamiento del territorio a nivel local.

Ante este panorama y la situación actual de los efectos del cambio climático a nivel global, se puede considerar como un elemento que consigue variar el patrimonio territorial de un destino turístico debido a los cambios naturales y culturales futuros, que a su vez podrían cambiar su puesta en valor ante la demanda turística.

Al respecto, Scott et al (2012) clasifican los impactos del cambio climático en los destinos turísticos como directos e indirectos (figura 2). Entre los primeros se encuentran los inviernos y veranos cálidos, la variación en los patrones de las precipitaciones y un aumento en los eventos extremos. En el caso de los impactos indirectos se asocian con cambios ambientales como la pérdida de biodiversidad; cambios en los patrones de movilidad del turista y socioeconómicos a nivel regional y global vinculados a los daños causados en los sectores económicos asociados con la actividad turística.

A partir de los estudios realizados por los Grupos de Trabajo del IPCC, la OMT y el Programa de las Naciones Unidad para el Medio Ambiente (2008) identifican las regiones turísticas más vulnerables por su localización geográfica (zonas tropicales, subtropicales, polares y con influencia marítima), donde las condiciones climáticas históricas se verán alteradas por cambios en el sistema climático (mapa 1), que causan efectos negativos (daño a infraestructuras, redistribución de flujos de visitantes, costos de operación más altos, pérdida de competitividad del destino), en la actividad turística y, por consiguiente, en su población y crecimiento económico. 
Figura 2: Impactos y efectos del cambio climático en destinos turísticos

- Impactos climáticos directos:

- Inviernos y veranos cálidos.

- Variación en las precipitaciones.

- Aumento de eventos extremos.

- Cambios ambientales indirectos:

- Pérdida de biodiversidad.

- Aumento en el nivel del mar.

- Enfermedades.

- Impactos en la movilidad:

- Costos del transporte y elección del destino.

- Impactos socioeconómicos indirectos:

- Impactos económicos regionales y globales.

- Aumento de riesgos sociales y gubernamentales.

\section{Efectos esperados}

- Cambios en los patrones de la demanda.

- Disminución en el suministro y baja calidad del agua.

- Daños en infraestructura turística.

- Pérdida de recursos turísticos.

- Riesgos a la salud de turistas y población local.

- Daños en sectores asociados al turismo.

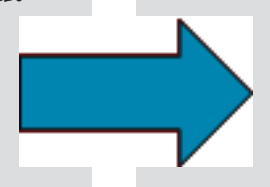


Mapa 1: Impactos del cambio climático en regiones turísticas a nivel mundial

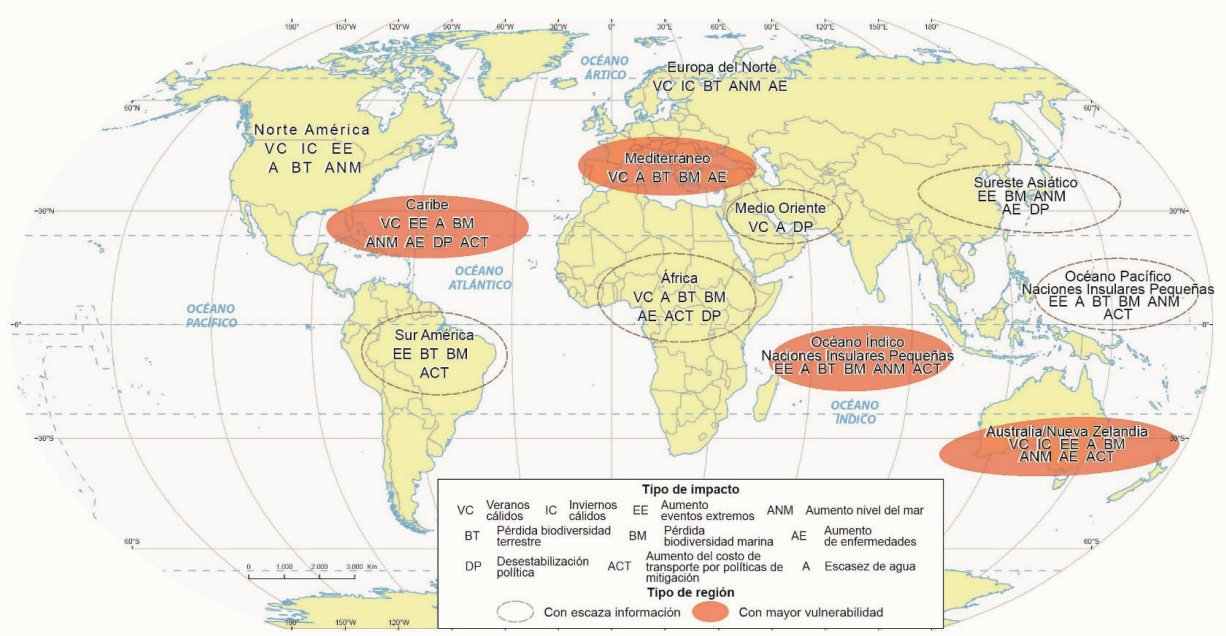

Fuente: OMT- PNUMA, 2008

\section{Características generales del cantón de Carrillo}

Con unas coordenadas de $10^{\circ} 28^{\prime} 59^{\prime \prime}$ latitud norte y $85^{\circ} 35^{\prime} 31^{\prime \prime}$ longitud oeste, y fundado en 1877, el cantón de Carrillo cuenta con un área de $577 \mathrm{~km}^{2}$, distribuidos en cuatro distritos: Filadelfia, Palmira, Sardinal y Belén (mapa 2). Colinda al norte el cantón de Liberia y este con el cantón de Bagaces, al sur con el cantón de Santa Cruz y al oeste con el océano Pacífico (Chinchilla, 1984 y Municipalidad de Carrillo, 2016). 
Mapa 2: Cantón de Carrillo, mapa general de localización

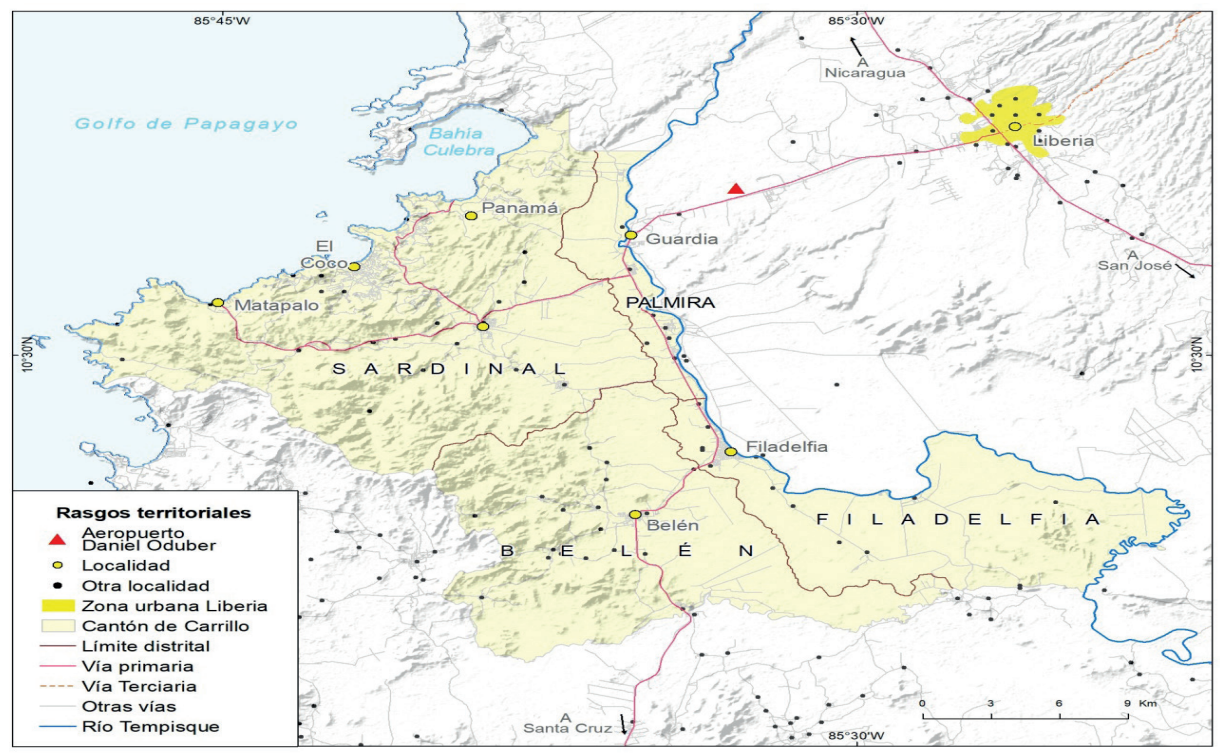

Fuente: elaboración propia a partir del Atlas ITCR, 2014

\section{Aspectos naturales}

Geológicamente el cantón de Carrillo está formado por materiales del Cretácico, Terciario y Cuaternario donde se identifican cinco subunidades geológicas: Complejo de Nicoya, Intrusivo del Complejo de Nicoya, Formación Barra Honda, Formación Bagaces y depósitos aluviales, coluviales y costeros recientes (Sprechmann, 1984).

El cantón presenta cinco unidades geomorfológicas (Bergoeing, 1998 y Madrigal et al, 1980), denominadas Formas de Sedimentación Aluvial, Denudación, Origen volcánico, Rocas Ígneas y sedimentarias marinas que se describen a continuación. Basado en estas cinco unidades se identifican tres unidades de relieve las cuales delimitan de forma clara el desarrollo de la actividad turística de la zona, la primea asociada a la llanura aluvial del río Tempisque en la cual se establecen las principales actividades agroindustriales y administrativas, la segunda con las serranías cercanas a la costa y en la cual se han establecido actividades pecuarias y se encuentran las principales áreas de bosque tropical seco y 
las mejores vistas al océano Pacífico por lo que son muy valoradas para el desarrollo inmobiliario asociado a la actividad turística, por último las áreas costeras las cuales han dado pie al turismo de sol y playa característico en el cantón.

Aunque en el área de estudio, según la clasificación de Holdridge, se ubican dos zonas de vida: bosque seco tropical y bosque húmedo premontano (Hartshorn, 1991); se localizan siete áreas silvestres protegidas: Parque Nacional Palo Verde, Refugio Nacional de Vida Silvestre Zapandi, Humedal Lacustrino Río Cañas, Refugio Nacional de Vida Silvestre Iguanita, Refugio Nacional de Vida Silvestre Riu, Refugio Nacional de Vida Silvestre Costa Esmeralda y Refugio Nacional de Vida Silvestre Hacienda El Viejo, administradas por el Área de Conservación Tempisque y el Área de Conservación Arenal Tempisque. Aunque a nivel nacional las áreas protegidas son uno de los recursos más visitados por los turistas, en el cantón es un destino no se consideran atractivos importantes para su visita por parte de visitantes extranjeros, a excepción del Parque Nacional Palo Verde; más bien son zonas de visitación para los residentes del cantón y áreas vecinas

Por su localización en el Pacífico Norte del país, el cantón de Carrillo presenta un clima tropical seco (Aw) según la clasificación de Köppen-Geiger, con el fin de caracterizar, con más detalle, las condiciones climáticas del cantón de Carrillo se utilizaron los registros entre los años 1957 y 2012 de la Estación Meteorológica Liberia, ubicada en el Aeropuerto Internacional Daniel Oduber (Villalobos et al, 2013). A partir de su análisis de los datos meteorológicos se identifica que la zona de estudio presenta una estación seca bien definida (figura 3), que va de diciembre a abril con promedios mensuales inferiores a los $60 \mathrm{~mm}$ y temperaturas medias entre los $26^{\circ} \mathrm{C}$ y $29^{\circ} \mathrm{C}$, periodo que coincide con la temporada alta de turistas y fundamentales para el turismo de sol y playa.

El periodo lluvioso se divide en dos partes, la primera incluye los meses de mayo, junio y julio, donde los primeros dos meses presentan precipitaciones superiores a los $200 \mathrm{~mm}$, por su parte el mes de julio muestra una disminución significativa con un promedio de $160 \mathrm{~mm}$, el cual coincide con la canícula y el periodo de vacaciones de medio año para el turismo nacional y de fin de curso para Norteamérica aumentando la visitación durante este mes. Por su parte la temperatura disminuye con respecto al mes de abril, con promedios entre $28^{\circ} \mathrm{C}$ y $26.7^{\circ} \mathrm{C}$. La segunda parte del periodo lluvioso abarca los meses de agosto a noviembre, donde el primero presenta un promedio de $228 \mathrm{~mm}$ aumento 
significativo con respecto al mes de julio, seguido del mes de septiembre y octubre con los promedios más altos del año $367.8 \mathrm{~mm}$ y $329.8 \mathrm{~mm}$ respectivamente.

Figura 3: Climograma estación meteorológica Liberia, 1957-2012

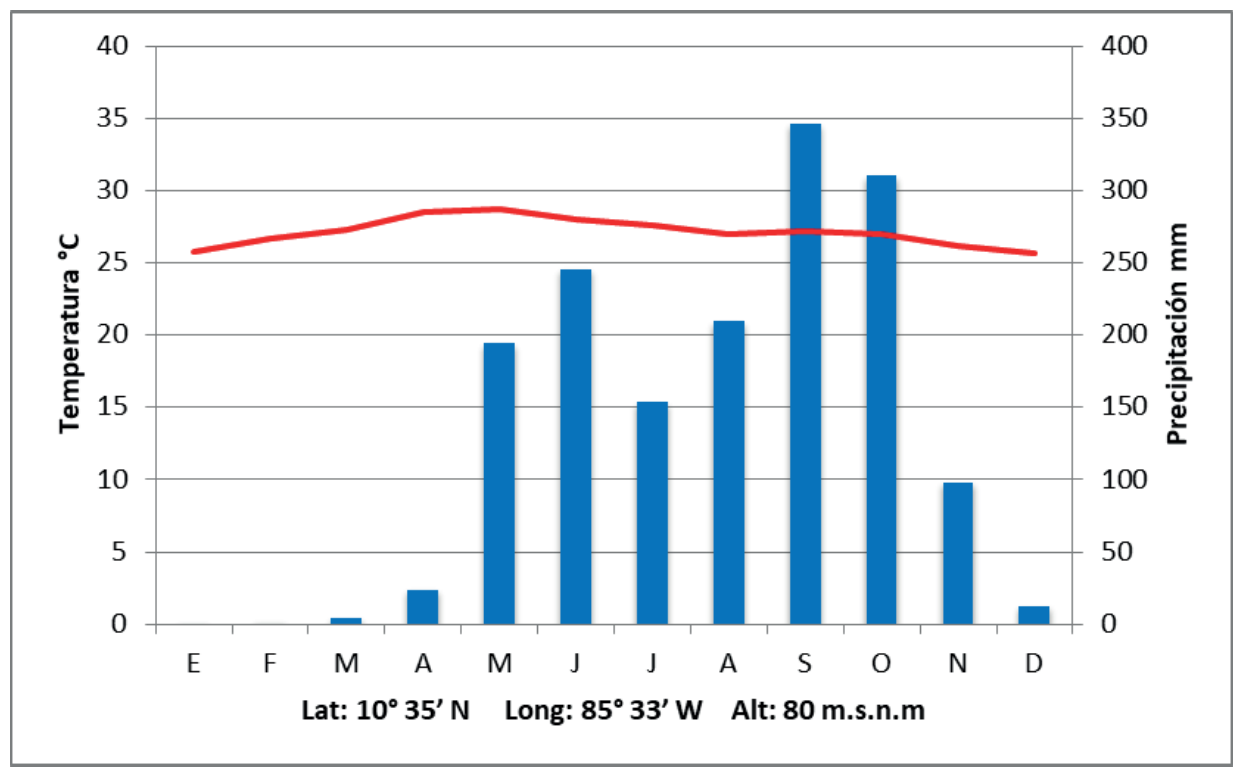

Fuente: elaboración propia a partir de Villalobos, 2013.

\section{Aspectos socioeconómicos}

Según el Censo Nacional de Población del año 2011, el cantón tenía una población de 37222 habitantes, con una tasa de crecimiento, para el periodo 2000-11, de 2,8\%, que lo ubica en el tercer lugar a nivel nacional. Aunque se localiza en la provincia de Guanacaste, la segunda con mayor población rural del país, el cantón de Carrillo presenta una población urbana del 62\%, asentada en los principales centros poblados como: Filadelfia (cabecera del cantón), Palmira y Sardinal, que es el más cercano a los desarrollos turísticos de sol y playa (Coco, Ocotal, Playa Hermosa y Playa Panamá) y en el cual habita un porcentaje muy alto de los trabajadores de estos centros turísticos.

Por su localización y condiciones físico-geográficas las principales actividades económicas del cantón son: agricultura (caña de azúcar, arroz, Melón), 
turismo (hotelería, tour operadores, turismo ecológico) y el comercio en general (empresas de servicios públicas y privadas).

Para el año 2011 la población económicamente activa del cantón de Carrillo era de 12646 personas lo que representa en términos porcentuales una tasa de ocupación del 43.9\%. La población ocupada (Tabla 1), se concentra en el sector terciario, donde destaca el distrito de Sardinal (77\%), en el cual se encuentra la mayor parte de la oferta turística del cantón y el distrito de Belén $(70,9 \%)$ que es punto de entrada al sector turístico de Tamarindo, localizado en el cantón vecino de Santa Cruz. La población ocupada en el sector secundario se concentra en el distrito de Palmira (22.8\%), debido que en este sector se localiza la mayor agroindustria del cantón asociada al cultivo comercial de la caña de azúcar; en el caso del sector primario se distribuye de forma regular en el cantón y se relaciona con cultivos de melón, sandía, arroz y actividades pecuarias.

Tabla 1: Cantón de Carrillo: distribución porcentual de la población ocupada por distrito, 2011

\begin{tabular}{|c|c|c|c|}
\hline Distrito & Sector primario & $\begin{array}{c}\text { Sector } \\
\text { secundario }\end{array}$ & Sector terciario \\
\hline Filadelfia & 19.1 & 19.3 & 61.6 \\
\hline Palmira & 13.7 & 22.8 & 63.5 \\
\hline Sardinal & 10.2 & 12.8 & 77 \\
\hline Belén & 14.2 & 14.8 & 70.9 \\
\hline $\begin{array}{c}\text { Total del } \\
\text { Cantón }\end{array}$ & 13.4 & 16 & 70.6 \\
\hline
\end{tabular}

Fuente: elaboración propia a partir de INEC, 2011; SEPSA, 2015.

El cantón sigue las tendencias socioeconómicas nacionales, por la distribución y características de la población, especialmente por el cambio en las actividades económicas y la tercerización de la economía a partir del desarrollo de la actividad turística, fenómeno que en el año 2016, según el registro al mes de julio de la oficina de patentes de la Municipalidad (Tabla 2 ), se encontraban registrados un total de 84 establecimientos de hospedaje, situación que denota un aumento en la oferta de hospedaje a pesar de las limitaciones en los permisos de construcción por parte del municipio a raíz de la disposición de agua potable a partir del año 2010 . 
Tabla 2: Cantón de Carrillo: oferta turística por distrito, 2016

\begin{tabular}{|c|c|c|c|}
\hline Distrito & Hospedaje & Alimentación & Entretenimiento \\
\hline Filadelfia & 6 & 13 & 21 \\
\hline Palmira & 4 & 11 & 10 \\
\hline Sardinal & 67 & 109 & 76 \\
\hline Belén & 5 & 9 & 23 \\
\hline Total cantón & 84 & 142 & 130 \\
\hline
\end{tabular}

Fuente: elaboración propia a partir de Municipalidad de Carrillo, 2016.

Además existen 142 establecimientos de alimentación y 130 establecimientos de entretenimiento. La mayor parte de la oferta se encuentra localizada en el distrito de Sardinal (71\%) donde se concentran los desarrollos turísticos del área de estudio, seguido de Filadelfia cabecera del cantón con un 11\% y por último el distrito de Palmira con un $7 \%$.

Por sus características naturales y culturales, el cantón de Carrillo, es un espacio con un gran potencial agrícola, pecuario y turístico; este último, principalmente por sus características climáticas, de relieve y vegetación las cuales son de gran atractivo para los visitantes los cuales desde el año 2001 al 2016 han presentado una variación significativa en la llegada al aeropuerto Daniel Oduber (tabla 3), pasando de 37.801 visitante durante el año 2001 a 572.544 para el año 2016 con una tasa de crecimiento para el periodo de $18 \%$, superior a la tasa del principal aeropuerto del país (Juan Santamaría), localizado en el área metropolitana que presentó un crecimiento de $4 \%$ para el mismo periodo. Aunque se el país de origen de los visitantes se ha diversificado, América del Norte y Europa se mantienen como las principales zonas emisoras desde el año 2001, dato que coincide con las estadísticas a nivel nacional. 
Tabla 3: Aeropuerto Daniel Oduber: Llegada de turistas por región de origen, 2001-16

\begin{tabular}{|c|c|c|c|c|}
\hline Región & $\mathbf{2 0 0 1}$ & $\mathbf{2 0 0 6}$ & $\mathbf{2 0 1 1}$ & $\mathbf{2 0 1 6}$ \\
\hline América del Norte & 35527 & 154938 & 242410 & 523362 \\
\hline América Central & 367 & 505 & 1283 & 1671 \\
\hline Caribe & 16 & 113 & 243 & 817 \\
\hline América del Sur & 386 & 595 & 1317 & 4168 \\
\hline Europa & 1358 & 4599 & 16702 & 37043 \\
\hline Asia & 101 & 567 & 1490 & 3119 \\
\hline África & 14 & 105 & 151 & 584 \\
\hline Otras zonas & 32 & 415 & 569 & 1794 \\
\hline Total & 37801 & 161837 & 264165 & 572544 \\
\hline
\end{tabular}

Fuente: elaboración propia a partir de ICT, 2016.

Basado en los datos de la Encuesta aérea de no residentes aplicada por el Instituto Costarricense de Turismo (ICT) durante el año 2016 en el aeropuerto Daniel Oduber, la estadía media de los turistas es de ocho noches, aunque los europeos son los visitantes que permanecen mayor tiempo en el país con un promedio de 13,1 noches seguido de los canadienses con 10,7, por su parte el gasto promedio fue de $\$ 885$, siendo los canadienses quienes más gastaron con un promedio de $\$ 899$ durante su visita. Con respecto a los motivos del viaje el $97 \%$ fue por vacaciones y solamente un $1 \%$ por negocios o motivos profesionales, indicando que habían estado en el país un promedio de 5 veces, aunque el $80 \%$ de los entrevistados indicaron que era la primera vez que visitaban el país principalmente por recomendación de amigos y familiares (66\%).

Por grupos de edad el 52\% de los encuestados se encuentran entre los 25 y 44 años, siendo un segmento de personas jóvenes y que viajan con niños y mayor poder adquisitivo y solamente el $2,7 \%$ de los visitantes se encuentran entre los 65 año y más, lo cual coincide con que el viaje lo realizaron un $54 \%$ en pareja, y el $30 \%$ con la familia y el $8 \%$ con amigos, de los cuales el $86 \%$ se hospedó en hoteles, un $14 \%$ en casa o condominio alquilado y un 1,7\% en casas y condominios propios, punto importante porque al relacionarlo con las con- 
secuencias producto del cambio climático en el área de estudio, afecta directamente a este grupo de la demanda. Por último, acorde con las características de la zona, las principales actividades realizadas durante el viaje fueron las de sol y playa con $90 \%$, tirolesa $46 \%$ y aguas termales con $36 \%$; por su parte la visita a comunidades rurales fue realizada solamente por el $3 \%$ de los visitantes y el $53 \%$ no visitó ningún área protegida.

\section{El patrimonio territorial del cantón de Carrillo}

A partir de fotografías aéreas del año 2017 del Instituto Geográfico Nacional de Costa Rica, se elaboró (según la metodología de Barrantes y Sandoval, 2016) el mapa de usos del suelo con el fin de identificar el patrimonio territorial del cantón de Carrillo (mapa 3). Para su estudio, los usos se clasifican en cuatro categorías a partir de las cuales se realiza el análisis sobre la relación de cada uno de los usos del suelo con el desarrollo de la actividad turística y su vinculación con el cambio climático.

Con el fin de tener una visión general del patrimonio territorial del área de estudio se presentan las principales características de la clasificación del uso del suelo:

- Pecuario: se incluyen el uso pecuario con pasto y el pecuario con árboles, los cuales se encuentran distribuidos en las zonas de pie de monte, algunas áreas de la parte alta de las serranías de los distritos de Sardinal y Belén y en las zonas de la llanura aluvial del Tempisque localizadas en los distritos de Filadelfia y Palmira.

- Agrícola: corresponde a los principalmente a los cultivos anuales como la caña de azúcar y el melón localizados en la llanura del río Tempisque en los distritos de Filadelfia, Palmira y Belén. En este caso es importante destacar el papel como patrimonio cultural y su papel en el desarrollo del cantón. En el caso de los cultivos permanentes se encuentra distribuido en pequeñas áreas del cantón.

- Forestal: dentro de esta categoría se encuentran las áreas silvestres protegidas del cantón y se incluyen el uso forestal - caducifolio que se localiza en las áreas montañosas de los distritos de Sardinal y Belén, el uso forestal - siempre verde correspondiente a pequeños parches de bosque en el pie de monte y algunas zonas de llanura en las 
comunidades de Belén y Filadelfia principalmente, por último el uso de humedal - manglar localizado en la zona de bahía Culebra y colindante con las áreas planificadas para desarrollo turístico del Polo turístico Papagayo.

- Urbano: se toman en cuenta el uso de suelo urbano y el de terreno descubierto, este último debido a que corresponde a proyectos inmobiliarios turísticos que se encuentran en construcción. Por su parte, el uso urbano se concentra en la zona costera del cantón, formando un corredor que se extiende desde playa Hermosa hasta playa Ocotal, también se encuentran de forma aislada algunos centros poblados que corresponden a las cabeceras de los distritos del cantón como Filadelfia, Belén, Palmira y Sardinal.

A pesar de la sequía que se presentó en el Pacífico Norte de Costa Rica a partir del año 2014 efecto del fenómeno del Niño y asociado al cambio climático que endureció el otorgamiento de los permisos de construcción por parte del municipio, el desarrollo inmobiliario no disminuyó en el área de estudio, por el contrario para el año 2017 se identifican un total de 280,76 hectáreas para un crecimiento del $18,73 \%$ con respecto al año 2013.

Durante este periodo, el uso del suelo con vocación turística se presenta en la zona costera y al lado de las principales vías de acceso a los desarrollos turísticos; para este año sigue creciendo la zona alrededor de El Coco, Ocotal y Playa Hermosa, por su parte, en el sector de Matapalo se consolida al construirse una segunda etapa del hotel Riu, el Riu Palace los cuales quedan aislados de las comunidades vecinas y se promocionan con servicio todo incluido siendo muy atractivos para los turistas en general. 
Mapa 3: Cantón de Carrillo, mapa de usos del suelo, 2017

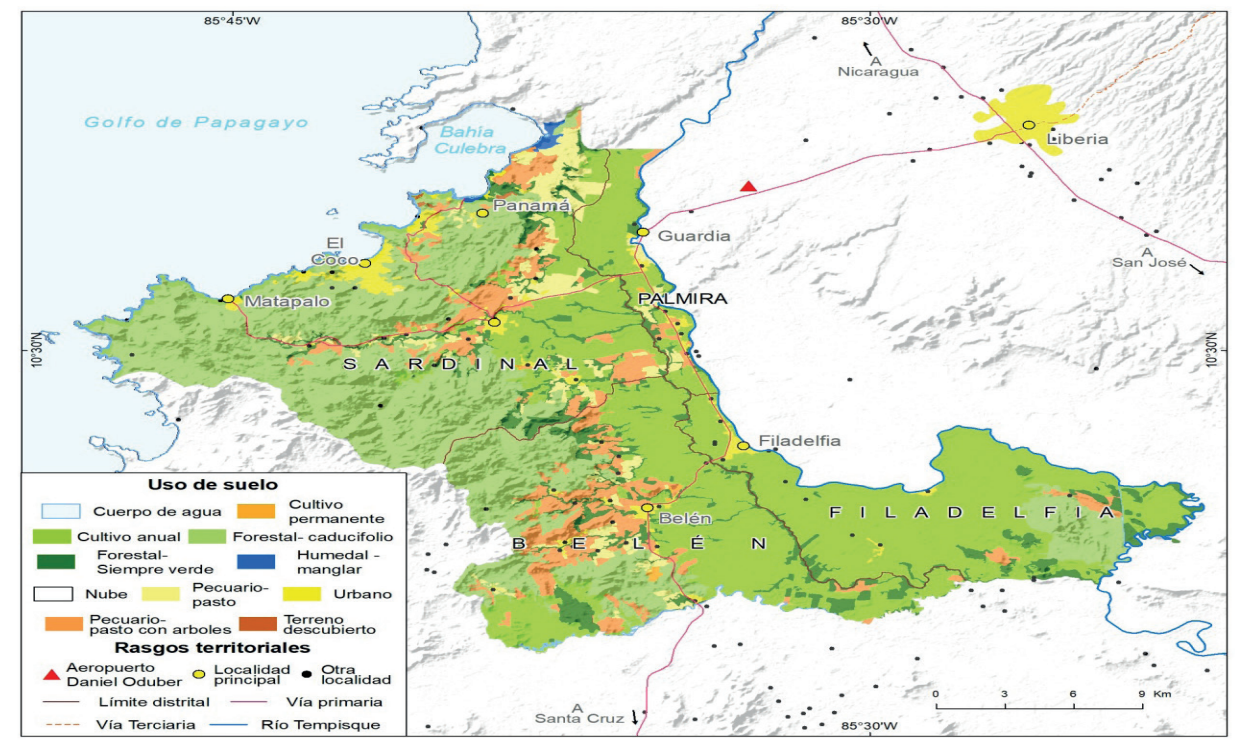

Fuente: Elaboración propia a partir de IGN, 2017.

En el caso del Polo Turístico Papagayo, aparecen sectores con uso del suelo en la categoría de preparación para construcción con un total de 46,19 hectáreas en el sector de punta Buena para el desarrollo de complejos hoteleros y residenciales; en este punto es importante resaltar que el principal efecto causado por el cambio climático es la disminución de la disponibilidad de agua potable en el cantón, el cual repercute de forma importante en los planes de expansión del polo turístico al paralizarse nueve concesiones al no poder comprobar la disponibilidad del recurso hídrico para su funcionamiento, que incluyen once hoteles y tres condohoteles con más de 800 habitaciones y una inversión superior a los $\$ 320$ millones (La Nación, 2017).

Por otra parte y como se demuestra en el mapa, la cobertura forestal de bosque siempre verde y especialmente del bosque seco tropical son uno de los principales recursos del cantón, por ser uno de los principales reductos de este tipo de bosque en Centroamérica y por las especies de flora y fauna que pueden encontrarse en el mismo, tomando en cuenta su vulnerabilidad durante la estación seca uno de los principales peligros son los incendios forestales y en charrales los cuales según los registros del Cuerpo de Bomberos de Filadelfia han 
disminuido en los últimos años pese a la sequía provocada por el fenómeno del Niño. En el caso de los incendios forestales se asocian principalmente a los siniestros que se dan en áreas protegidas y zonas boscosas importantes, los cuales presentan un aumento en el periodo 2012-15 a partir del cual disminuyen hasta llegar a cero durante los años 2016 y 2017, según los bomberos consecuencia de la toma de conciencia por parte de las empresas del sector y las comunidades locales organizadas mediante brigadas contra incendios.

De forma más detallada, Argos (2008) elabora un inventario de atractivos turísticos para la Subunidad de Planeación Guanacaste Norte, mediante el cual identifica en el cantón de Carrillo un total de 52 atractivos distribuidos en cinco categorías (tabla 4). De éstos, el 78\% corresponde a sitios naturales sobresaliendo las playas, islas y montañas; la categoría de museos y manifestaciones culturales con el $12 \%$, corresponde a ruinas y lugares arqueológicos; en caso de folclore y acontecimientos programados, con $4 \%$ cada uno de ellos, los atractivos identificados se relacionan con fiestas patronales, corridas de toros y vida nocturna; por último, la categoría de realizaciones técnicas, con un $2 \%$, se asocia con explotaciones agropecuarias relacionadas con el cultivo de la caña de azúcar.

Tabla 4: Cantón de Carrillo, clasificación de atractivos turísticos

\begin{tabular}{|c|c|}
\hline Categoría & Cantidad \\
\hline Sitios naturales & 41 \\
\hline Museos y manifestaciones culturales & 6 \\
\hline Folclore & 2 \\
\hline Realizaciones técnicas & 1 \\
\hline Acontecimientos programados & 2 \\
\hline Total & 52 \\
\hline
\end{tabular}

Fuente: Argos, 2008.

Por las condiciones del desarrollo de la actividad turística en el área de estudio, prevalece el turismo de sol y playa, en el cual los principales recursos turísticos están asociados a la zona costera, de gran atractivo para la demanda nacional y internacional.

Con respecto a los instrumentos de ordenamiento del territorio establecidos a nivel nacional, el cantón de Carrillo, no posee un plan regulador vigente 
aunque se realizó una propuesta del mismo en el año 2010 que no cumplió con los requisitos establecidos para su aprobación, en esta propuesta se aborda la actividad turística de forma superficial a pesar de ser una de las principales actividades económicas de la zona de estudio y no hace referencia al cambio climático cuyo impacto por el fenómeno del Niño en la zona se conoce desde hace más de dos décadas.

En este caso de los planes reguladores costeros en el cantón de Carrillo se ha realizado el plan regulador integral para todas las playas del municipio, aunque hay que resaltar que los planes más antiguos se realizaron de forma individual y aislada dando como resultado una planificación territorial fragmentada que refleja una visión puramente comercial por parte de los desarrolladores encargados de las concesiones turísticas en dichos lugares.

\section{Influencia del cambio climático en el área de estudio}

Según los datos de la estación meteorológica Llano Grande, del año 1965 al año 1969, se presentaron precipitaciones inferiores a los promedios anuales, coincidiendo con un periodo de sequía similar al presentado a partir del año 2013 y el cual recuerdan muchos habitantes del cantón, en este periodo el año 1965 fue el más seco con un promedio de $75.4 \mathrm{~mm}$.

Durante el periodo de 1977 a 2010 (figura 4), se presentaron diversos eventos asociados a la variabilidad climática del área de estudio; en el caso de la temperatura no se presenta una variación importante, donde la temperatura inferior se presenta en el año 1999 con un promedio de $26.4^{\circ} \mathrm{C}$ y la superior en el año 1997 con una media de $27.9^{\circ} \mathrm{C}$. En el caso de las precipitaciones se presenta una variación significativa durante el periodo, destacan los años 1977 , 1987, 1997 y 2007 con las precipitaciones más bajas, siendo el año 1977 el más seco con un promedio anual de $82 \mathrm{~mm}$; estos coinciden con años en los que se presentó el fenómeno del Niño en el país.

Por otra parte, los años 1996, 1999, 2008 y 2010 son los años con mayores precipitaciones del periodo, en el cual 1999 es el año más lluvioso con un promedio de $229.4 \mathrm{~mm}$, estos años coinciden con el fenómeno de la Niña en el país y en los cuales se presentaron eventos que causaron inundaciones y pérdidas en infraestructura en la zona de estudio. 
Figura 4: Temperatura y precipitación promedio anual estación meteorológica Llano Grande, 1977-2010

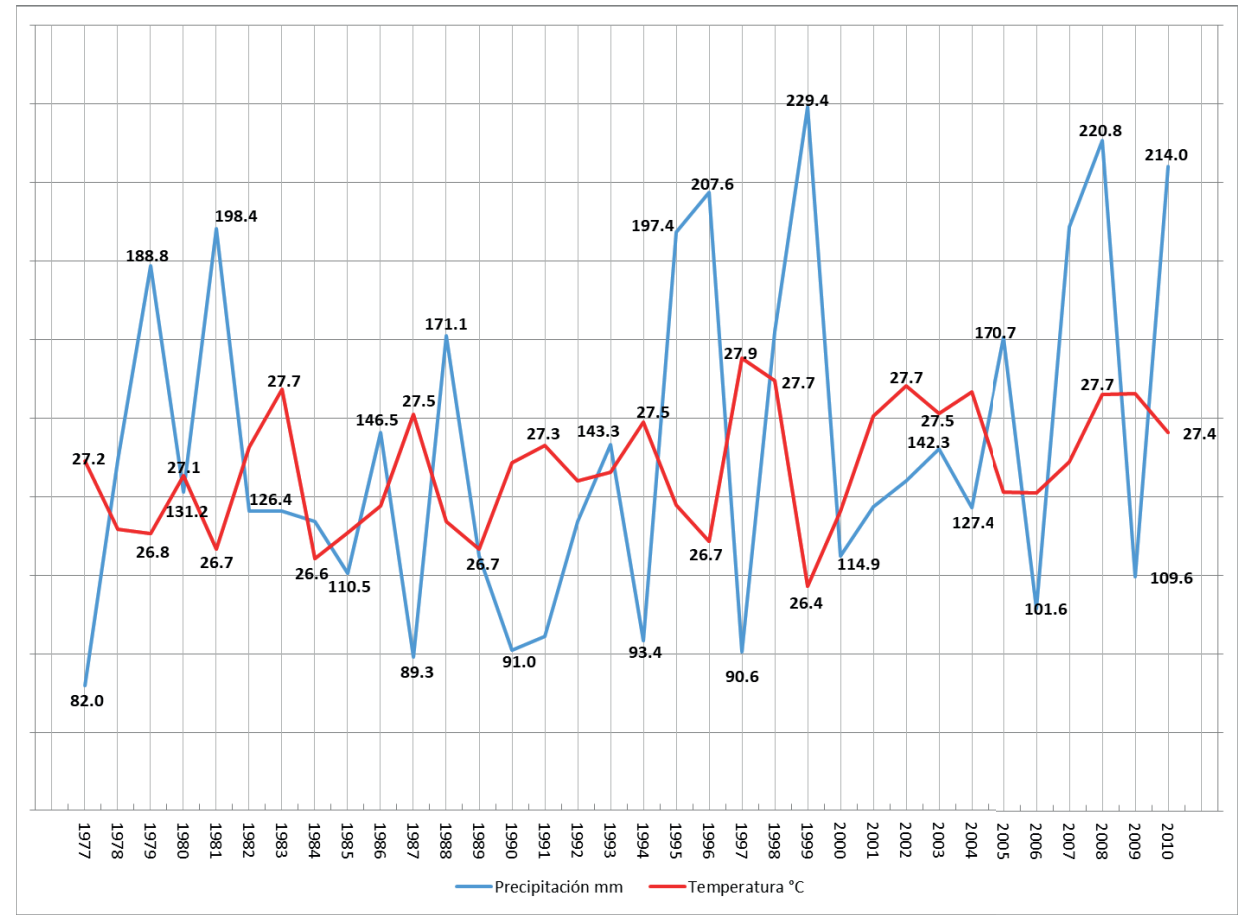

Fuente: elaboración propia a partir de datos del IMN.

Con respecto a los promedios anuales de precipitación en el periodo 1998-2015 registrados en la estación meteorológica Aeropuerto Liberia oeste 07, se observa una disminución en las precipitaciones a partir del año 2009. En este intervalo, los años 2009, 2014 y 2015 son los años con menor precipitación, donde el último presenta el menor promedio anual con $59.6 \mathrm{~mm}$, el más seco desde el año 1960 según los registros del Instituto Meteorológico Nacional para las dos estaciones estudiadas. Este periodo coincide con el inicio de la sequía en el Pacífico Norte del país la cual causó un impacto significativo en el suministro de agua potable en las comunidades del cantón de Carrillo, especialmente en las zonas costeras donde se salinizaron los acuíferos de El Coco y Playa Hermosa, sectores donde se ubican los principales centros turísticos de la zona. 


\section{Impactos del cambio climático en el patrimonio territorial del cantón de Carrillo}

La valoración de los efectos del cambio climático en un destino turístico no es simple, tomando en cuenta que como sistemas complejos se encuentran conformados por múltiples variables en interacción. En este caso al tomarse en cuenta los usos del suelo para identificar y clasificar el patrimonio territorial del área de estudio y al estar vinculados con el impacto del cambio climático en la actividad turística, el análisis se centra en las zonas de mayor crecimiento turístico del cantón.

Es importante resaltar que el principal impacto por el cambio climático en el cantón son los fenómenos del Niño y la Niña que tienen entre sus principales efectos periodos de altas o bajas las precipitaciones fuera de los valores promedio de la zona que afectan de forma diferenciada las áreas vulnerables del cantón y la evolución de la actividad turística. Razón por la que la investigación se desarrolló durante el periodo de sequía causado por el fenómeno del Niño entre los años 2013 y 2015

En el caso de los distritos de Palmira y Belén, los impactos causados por el cambio climático son menores porque no se identificó afectación importante en los recursos turísticos, la oferta y demanda turística, a pesar de lo anterior es necesario resaltar que la principal vía de articulación entre las ciudades de Liberia y Santa Cruz además de su conexión con el aeropuerto Daniel Oduber (principal punto de ingreso del turismo internacional a las zonas turísticas de sol y playa de la región), se extiende a lo largo de ambos distritos y es vulnerable ante las inundaciones causadas por el río Tempisque, hecho que impacta de forma directa la actividad turística y la población de estos sectores. Los principales efectos identificados se asocian con las comunidades locales y las políticas públicas las cuales se relacionan debido a que no existe un plan regulador cantonal que permita una adecuada planificación del territorio, además la falta de agua potable que afecta las comunidades y la actividad turística se ha visto incrementada por la mala gestión y la falta de coordinación entre las instituciones estatales en sus diferentes niveles.

Por su parte, los impactos identificados en el distrito de Filadelfia se relacionan principalmente a su condición de cabecera cantonal y por estar localizada en la llanura del río Tempisque, la población, oferta turística e infraestructura como la carretera que une Liberia y Santa Cruz es vulnerable a las 
inundaciones que se presentan por eventos extremos durante la época de lluvias como temporales y huracanes, durante la época seca el distrito es afectado por altas temperaturas las cuales junto con las malas prácticas de los pobladores y agroindustrias aumentan la vulnerabilidad ante los incendios en los cultivos de caña de azúcar y los asociados a la preparación de terrenos para el cultivo (roza y quema) presentes entre los meses de enero y abril, coincidiendo con la época de mayor afluencia de visitantes a la zona. Al igual que los distritos de Palmira y Belén los principales efectos se identifican en los aspectos relacionados con la comunidad local por la disponibilidad del agua potable y las políticas públicas puntualmente por la falta de planificación territorial y la inadecuada gestión pública ante los problemas asociados a la sequía.

El distrito de Sardinal por sus características geomorfológicas presenta una alta vulnerabilidad ante deslizamientos e inundaciones que pueden afectar directamente las vías que comunican el poblado de Sardinal (principal centro distribuidor de la demanda turística en el cantón) con las zonas de desarrollo turístico de sol y playa como Matapalo, Ocotal, El Coco, Hermosa y Panamá las cuales por ser zonas formadas por material poco consolidado son vulnerables ante la erosión causada por las mareas altas, las precipitaciones y el aumento de la escorrentía superficial asociada al crecimiento de la urbanización en los últimos cinco años. Por su parte, los efectos de la sequía causados por el fenómeno del Niño en los recursos turísticos se vinculan con la flora y la fauna de la zonas boscosas localizadas en las laderas de las áreas con mayor plusvalía por sus panorámicas al océano Pacífico que son vulnerables a incendios forestales durante la época seca, situación que pone en riesgo a la población local, los turistas e infraestructura turística.

En el distrito se localiza la mayor parte de la oferta del cantón la cual se ha visto afectada de forma importante por la sequía y la disponibilidad de agua potable, más que todo para las pequeñas y medianas empresas que dependen de la red de distribución pública y que no poseen pozos propios o en concesión como sucede con hoteles grandes y algunas áreas residenciales. Como en los distritos anteriores las variables relacionadas a las políticas públicas y la comunidad local son las que presentan mayores efectos, en este último caso aquellos vinculados a la sequía causaron mayores consecuencias en la población debido a que muchos de los pozos familiares (de poca profundidad) se secaron, además por la disminución del nivel de los acuíferos que suministraban el agua potable a las comunidades de El Coco y Hermosa, se presentó un proceso de salinización dejando 
sin el servicio no solo a los habitantes de están comunidades sino también a la mayor parte de las actividades económicas vinculadas con la actividad turística.

En el caso de las políticas públicas, los efectos son más amplios debido a que en la zona convergen tres ámbitos de planificación relacionados a la actividad turística y que no se encuentran vinculados de forma integral. En un primer nivel se encuentra el gobierno local que no posee un plan regulador que dirija la planificación territorial de la zona, vinculado a este mismo tema en un segundo nivel se encuentra la zona marítimo terrestre correspondiente a los 200 metros de costa a partir de la pleamar y en la cual se localizan los espacios en concesión para el desarrollo turístico y que por ley debe de tener un plan regulador y que actualmente no se encuentra vigente porque el nuevo plan se encuentra en proceso ante la sala Constitucional. En un tercer nivel se encuentra la zona que corresponde al Polo Turístico Papagayo el cual posee una legislación propia que lo desvincula de los niveles de planificación anteriores y es administrada directamente por el ICT el cual aunque en sus proyectos toma en cuenta la población de las comunidades localizadas en esta zona sus intereses principales van dirigidos a facilitar las inversiones correspondientes al desarrollo de los complejos turísticos planificados.

Basado en las unidades del relieve del cantón, las zonas vulnerables identificadas se encuentran ligadas a inundaciones asociadas al desbordamiento del río Tempisque en los distritos de Palmira y Filadelfia que afecta no solo a la población sino la carretera que comunica las ciudades de Liberia y Santa Cruz, considerada de primer orden y por la cual se desplaza la mayoría de los turistas que vistan la zona, también se presentan inundaciones en el valle del río Sardinal donde se localiza la vía de acceso a playa Matapalo donde se localiza uno de los principales centros turísticos del área. Sobre esta misma vía de comunicación se localizan zonas vulnerables a deslizamientos de material proveniente de las serranías cercanas por su elevada pendiente, hecho que también se identifica sobre las vías de comunicación que unen el poblado de Sardinal con las zonas turísticas de El Coco, Ocotal y Hermosa.

\section{Conclusiones}

El cambio en la definición del patrimonio de un elemento individual a un patrimonio integral reflejado en el concepto de patrimonio territorial permite la puesta en valor de los diferentes elementos naturales y culturales de las 
comunidades por parte de la sociedad contemporánea, aunque no queda exento de ser minimizado por el cambio en los gustos de la misma.

Esta visión holística del patrimonio territorial se convierte en una expresión del enfoque de la Geografía como ciencia, que tiene al espacio geográfico como objeto de estudio, que por su carácter complejo, se aborda de forma integral con el objetivo de beneficiar a la población por medio de propuestas de desarrollo más cercanas a la realidad de las comunidades locales.

Por el carácter dinámico de los elementos que componen el patrimonio territorial de una localidad y sujetos a la valoración social de la población, el cambio climático se convierte en una amenaza para conservar su estatus por parte de la población y pone en riesgo el desarrollo de la actividad turística de los territorios, siendo el ordenamiento territorial una herramienta que permite abordar de forma integral los recursos de las comunidades y proponer opciones de desarrollo sostenible y acordes con los cambios esperados a nivel local por el cambio climático.

El cantón de Carrillo al igual que la mayor parte de del país basa su producto turístico en los recursos naturales (playas, clima, vegetación, recurso hídrico) los cuales como se demuestra en el estudio realizado son susceptibles a los efectos del cambio climático a nivel local como los fenómenos del Niño y la Niña, lo cual pone en riesgo el desarrollo de las comunidades, especialmente al no existir un plan regulador cantonal que aborde de forma integral las problemáticas del territorio junto con el plan regulador costero vigente.

A nivel nacional existe poca información científica sobre los impactos del cambio climático en la actividad turística a nivel local, fundamentales para la toma de decisiones en la planificación territorial y administrativa de los gobiernos locales, instituciones públicas y empresas privadas, situación que puede ser revertida a partir de las investigaciones realizadas por parte de las universidades públicas con el fin de facilitar la toma de decisiones y acompañar a las comunidades locales por medio de alternativas que favorezcan su desarrollo. 


\section{Referencias}

ANTÓN, S. “La urbanización turística. De la conquista del viaje a la reestructuración de la ciudad turística”. Documents D'anàlisi Geográfica, n. 32, pp. 17-43, 1998.

ARGOS, T. Dinámica territorial del desarrollo turístico costero: Unidad Turística Guanacaste Norte. San José: CREST, 2008.

BARQUERO, M. “Falta de agua atasca proyectos por \$320 millones en Papagayo”. Periódico La Nación, pp. 23-24, 17 mar. 2017.

BARRADO, D. “El concepto de destino turístico: una aproximación geográfico-territorial”. Estudios Turísticos, n. 160, pp. 45-68, 2004.

BARRANTES, O., \& SANDOVAL, L. "Uso/cobertura de la tierra en los cantones de Upala, Guatuso y Los Chiles en el año 2011”. Revista Geográfica de América Central, n. 1, v. 56, 59-91, 2016. doi:http://dx.doi.org/10.15359/rgac.1-56.3.

BERGOEING, J. Geomorfología de Costa Rica. San José: Instituto Geográfico Nacional, 1998.

CHINCHILLA, J. Atlas cantonal. San José: IGN, 1984.

DOCTOR, A. M. "El itinerario como herramienta para la puesta en valor turístico del patrimonio territorial”. Cuadernos de turismo, n. 27, pp. 273-289, 2011.

ESTEBAN, A. et al. Turismo y cambio climático. Recuperado de http://www.gadeso. org/sesiones/gadeso/web/14_paginas_opinion/ca_10000191.pdf, 2007.

HARTSHOM, G. y EN JANZEN, D. (editor). Historia natural de Costa Rica. San José: Editorial de la Universidad de Costa Rica, 1991.

INSTITUTO COSTARRICENSE DE TURISMO. Encuesta Aérea de No Residentes, Aeropuerto Internacional Daniel Oduber Quirós. San José: ICT, 2015.

INSTITUTO NACIONAL DE ESTADÍSTICA Y CENSOS. X censo nacional de población: caracteristicas sociales y demográficas. San José: INEC, 2012. 
MADRIGAL, R. y ROJAS, E. Manual descriptivo del mapa geomorfológico del Costa Rica, escala 1: 200.000. San José: SEPSA, 1980.

MUNICIPALIDAD DE CARRILLO. Datos sobre entornosy demografia del cantón de Carrillo. Recuperado de https://www.municarrillo.go.cr/index.php?option=com_ content\&view=article\&id=204\&Itemid=710, 2016.

SEPSA. Boletin Estadistico Agropecuario, n. 25. San José: SEPSA, 2015.

SCOTT, D. et al. Tourism and climate change: Impacts, adaptation and mitigation. Routledge, 2012.

SPRECHMANN, P. Manual de geología de Costa Rica. San José: Editorial Universidad de Costa Rica, 1984.

TRUJILLO, G. F. “El patrimonio territorial en el plan de ordenación del territorio de Andalucía: indefiniciones y dificultades para un conocimiento preciso". BAGE, 63, 2013.

VALCÁRCEL, J. O. "El patrimonio territorial: el territorio como recurso cultural y económico”. Ciudades, 04, 31-48, 1998.

VILLALOBOS, R. Descripción del clima. Cantón de Liberia. San José: IMN, 2013.

WORLD TOURISM ORGANIZATION \& UNITED NATIONS ENVIRONMENT PROGRAMME. Climate Change and Tourism: Responding to Global Challenges. Madrid: World Tourism Organization, 2008. 